\title{
Comparison of diabetic retinopathy classification using fluorescein angiography and optical coherence tomography angiography
}

\author{
Mário Soares, ${ }^{1,2}$ Catarina Neves, ${ }^{1}$ Inês Pereira Marques, ${ }^{1}$ Isabel Pires, ${ }^{1,2}$ \\ Christian Schwartz, ${ }^{1}$ Miguel Ângelo Costa, ${ }^{1}$ Torcato Santos, ${ }^{1}$ Mary Durbin, ${ }^{3}$ \\ José Cunha-Vaz ${ }^{1,4}$
}

${ }^{1}$ AIBILI-Association for Innovation and Biomedical Research on Light and Image, Coimbra, Portugal

${ }^{2}$ Ophthalmology Department, Centro Hospitalar e

Universitário de Coimbra (CHUC), Coimbra, Portugal

${ }^{3}$ Advanced Development, Carl Zeiss Meditec, Inc, Dublin, California, USA

${ }^{4}$ University of Coimbra Coimbra, Portugal

\section{Correspondence to}

Mário Soares, AIBILI, Azinhaga de Santa Comba, Celas. Coimbra 3000-548, Portugal; mariosoares0001@gmail.com

Received 28 July 2016 Revised 13 October 2016 Accepted 15 November 2016
CrossMark

To cite: Soares $M$, Neves $C$, Marques $\mathrm{IP}$, et al. Br J Ophthalmol 2017;101:6268.

\begin{abstract}
Purpose To analyse and compare the classification of eyes with diabetic retinopathy using fluorescein angiography (FA) and optical coherence tomography angiography (OCTA) performed either with AngioPlex or AngioVue.
\end{abstract}

Methods This was an observational cross-sectional study of 50 eyes from 26 diabetic subjects. Two independent graders classified the FA angiograms, to assess the presence and severity of several characteristics according to the ETDRS Report 11, and a similar evaluation was performed for each $3 \times 3 \mathrm{~mm}$ OCTA image from the superficial retinal layer and for the full retina slab. Results Percentages of non-gradable images for the outline of foveal avascular zone (FAZ) in the central subfield (CSF) were $29.0 \%$ for FA, $12.0 \%$ for AngioVue and $3.0 \%$ for AngioPlex. For capillary loss, percentages of non-gradable images in the CSF were $25.0 \%$ for FA, $11 \%$ for AngioVue and $0.0 \%$ for AngioPlex. For the inner ring $(\mathrm{IR})$, percentages of non-gradable images were $12.5 \%$ for FA, $11.5 \%$ for AngioVue and $0.5 \%$ for AngioPlex. Agreement between graders was substantial for outline of FAZ. For capillary loss, the agreement was fair for the CSF, and moderate for the IR.

Conclusions The OCTA allows better discrimination of the CSF and parafoveal macular microvasculature than FA, especially for FAZ disruption and capillary dropout, without the need of an intravenous injection of fluorescein. In addition, FA had also a higher number of non-gradable images. The OCTA can replace with advantage the $F A$, as a non-invasive and more sensitive procedure for detailed morphological evaluation of central macular vascular changes.

Trial registration number NCT02391558,

Pre-results.

\section{INTRODUCTION}

The use of fluorescein angiography (FA) was first described in $1961^{1}$ and introduced as a widespread technique by Gass et $a l^{2}$ in 1967 . It has been the gold standard for evaluating vascular alterations that occur in different stages of diabetic retinopathy (DR). Those alterations have been evaluated in a systematic fashion, and a classification system was developed to assess severity of selected characteristics such as capillary loss and dilatation, size of foveal avascular zone (FAZ), arteriolar abnormalities and several other features based on the observation of the angiograms by the ETDRS Group. ${ }^{3}$ However, reproducibility of grading is still problematic ${ }^{4}$ and despite the additional information from FA, the Diabetic Retinopathy Study (DRS) and ETDRS still rely on the interpretation of stereo fundus photography ${ }^{5}$ for assessing severity scale and the need for laser photocoagulation. ${ }^{6}$ In addition, FA is invasive, involves risks and can cause nausea or allergic reactions. ${ }^{7}$ With the recent development of optical coherence tomography angiography (OCTA), ${ }^{8-17}$ which is based on the principle of identifying temporal changes of OCT signal, caused by flowing of the erythrocytes in blood vessels, a new method for visualisation of the retinal microvasculature emerged. This technique allows the construction of three-dimensional blood flow information, and therefore can serve as a noninvasive method to evaluate ocular circulation.

In this study, we aimed to compare gradings of vascular changes of eyes with DR, using FA and OCTA, following ETDRS Report 11 methodology.

The comparison focused on the superficial retinal layer, because an exact comparison cannot be made between both methods for the deeper layer, as the deep retinal layer is not distinguishable with FA. ${ }^{18}$ However, a comparison was also performed between FA and OCTA full retina slab.

\section{MATERIALS AND METHODS Participants}

The tenets of the Declaration of Helsinki were followed and informed consent to participate in the study was obtained from all subjects after all procedures were explained. This was an observational cross-sectional study. The study was performed at the Association for Innovation and Biomedical Research on Light and Image from September 2015 through December 2015 (http://www. clinicaltrials.gov NCT02391558) and included 26 patients with different levels of DR. Patients with any other chorioretinal disease, history of vitreous surgery or severe media opacity, such as dense cataract or vitreous haemorrhage, and high ametropic eyes (sphere dioptres $>6$ and cylinder dioptres $>3$ ) were excluded to ensure good quality images.

All the patients underwent a complete ophthalmological examination, including best-corrected visual acuity and intraocular pressure assessment, dilated fundus examination, 7-field colour fundus photography (CFP) (according to the ETDRS grading protocol), FA (Heidelberg Spectralis, Heidelberg, Germany) and OCTA (Zeiss Cirrus 5000 AngioPlex and Optovue RTVue AngioVue 
System). The values of haemoglobin A1c and duration of diabetes were recorded for analysis. ETDRS grade was determined from 7-field CFP (Topcon Medical Systems).

No patients with clinical macular oedema according to DRCR.net OCT definition $(\geq 305 \mu \mathrm{m}-$ males $/ \geq 290 \mu \mathrm{m}-$ females) for Cirrus high definition (HD)-OCT were included. FA was performed with the Spectralis Heidelberg retina angiograph+OCT (Heidelberg Engineering, Heidelberg, Germany) system, and clear and focused $20^{\circ}$ images of the early stage of the procedure were obtained. Patients were given a $3 \mathrm{~mL}$ intravenous injection of fluorescein solution.

\section{Optical coherence tomography angiography}

OCTA was performed using two different devices. The Optovue RTVue XR Avanti AngioVue software uses the split-spectrum amplitude decorrelation angiography algorithm. ${ }^{11}$ This instrument has a $70 \mathrm{kHz}$ axial scan repetition rate, using a light source centred on $840 \mathrm{~nm}$ and a bandwidth of $50 \mathrm{~nm}$. The optical axial resolution is $5 \mu \mathrm{m}$, and the transverse is $15 \mu \mathrm{m}$. Each OCTA volume contains $304 \times 304 \mathrm{~A}$-scans with two consecutive B-scans (M-B frames) captured at a fixed position before proceeding to the next sampling location. The volumes were registered and the B-scan images were compared to calculate the decorrelation in the images along with motion artefact removal.

The scanning area was captured in $3 \times 3 \mathrm{~mm}$ sections and was centred on the fovea.

The volume data gathered was segmented using the internal limiting membrane (ILM) as a plane of reference, with an inner boundary at $3 \mu \mathrm{m}$ beneath the ILM and the outer boundary was set at $15 \mu \mathrm{m}$ beneath the inner plexiform layer (IPL) in order to obtain images of the superficial retinal layer (SRL). An additional segmentation was manually performed for the whole neurosensory retina, generating a slab from the ILM to the retinal pigment epithelial (RPE) that includes both superficial and deep plexus.

The other instrument used for the OCTA images was based on the Zeiss Cirrus HD-OCT 5000. This instrument has a $68 \mathrm{kHz}$ axial scan repetition rate. The axial and transverse resolution in tissue is 5 and $15 \mu \mathrm{m}$, respectively. AngioPlex is a new technology based on the optical microangiography complex ${ }^{15} 16$ algorithm. AngioPlex uses amplitude and phase aspects of the OCT signal to extract in vivo blood flow information. The scans comprise a series of clusters of repeated B-scans, where each B-scan consists of 245 A-scans. Tracking on Cirrus is provided by registering fundus images provided by the line-scan ophthalmoscope (LSO) image. This image is inherently registered to the OCT data by using the same optical path. Images are acquired at a rate of $15 \mathrm{~Hz}$. Any OCT B-scans that were acquired during an LSO frame that is offset by $>30 \mu \mathrm{m}$ from the reference LSO frame are discarded and that region of the retina is re-acquired. ${ }^{19}$

The OCT volume scan had dimensions of $3 \times 3 \mathrm{~mm}$ and $2 \mathrm{~mm}$ deep. The averaged OCT B-scans were segmented to obtain the ILM and RPE. The inner retina was estimated as being the tissue between the ILM and an offset from the RPE of $110 \mu \mathrm{m}$. The SRL was defined as the inner $70 \%$ of the inner retina. A preset slab of the entire retina was also assessed for direct comparison with FA.

Low-quality images were graded conservatively, and considered non-gradable when the characteristic evaluated was not perceived. OCTA images were excluded from analysis if the
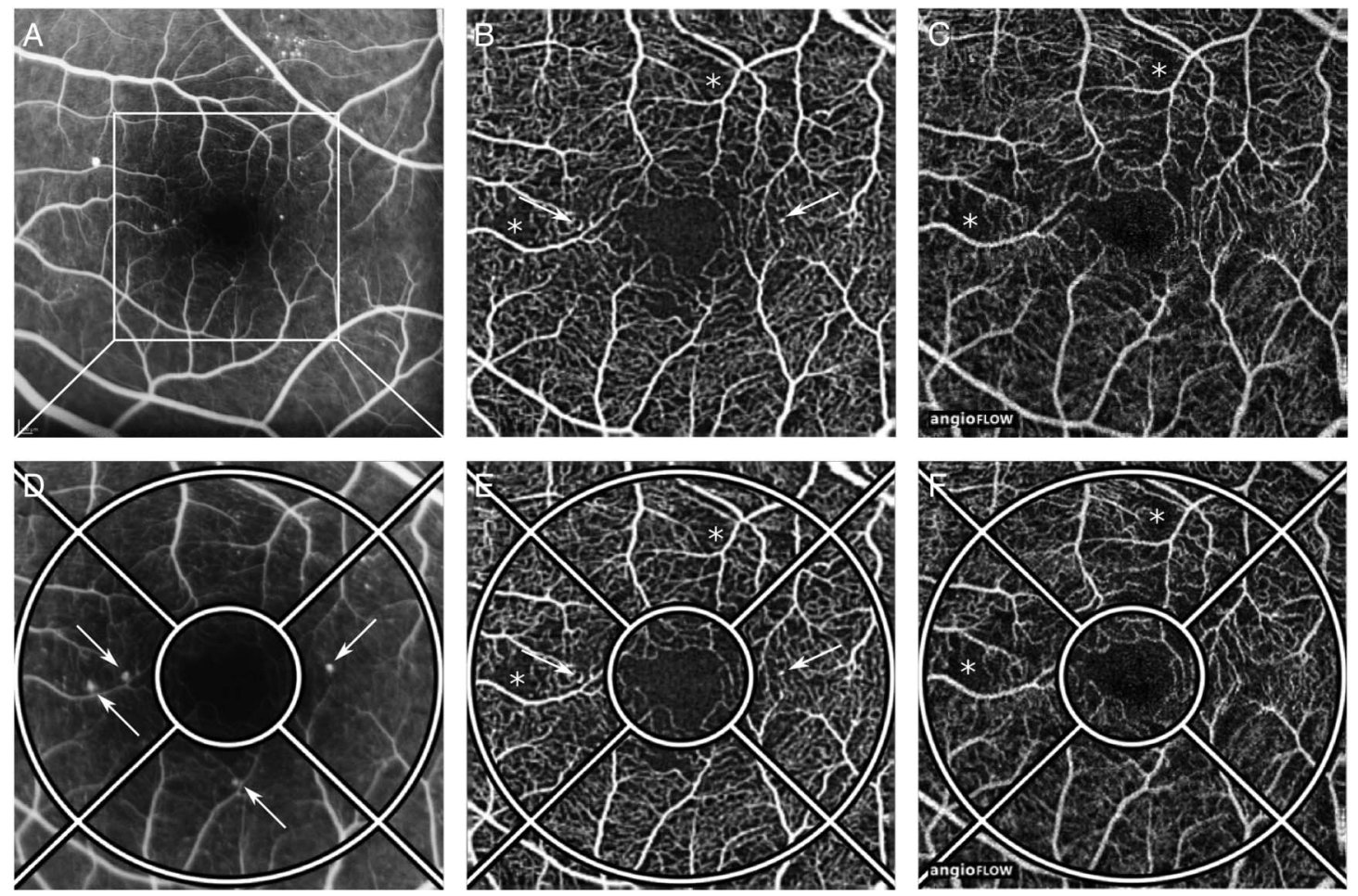

Figure 1 Comparison of fluorescein angiography (FA) with optical coherence tomography angiography (OCTA) images for the superficial retinal layer (AngioPlex and AngioVue). (A) Early $20^{\circ} \mathrm{FA}$ image. The white square outlines the area of the $3 \times 3 \mathrm{~mm}$ graded for this study. (B) $3 \times 3 \mathrm{~mm} 0 \mathrm{CT}$ angiogram obtained from Zeiss AngioPlex showing areas of non-perfusion (star) and microaneurysms (arrows). (C) $3 \times 3-\mathrm{mm}$ 0CTA from AngioVue showing the same areas of non-perfusion found for AngioPlex images, but with absence of microaneurysms. (D) Magnification of the $3 \times 3 \mathrm{~mm}$ square with grid overlaid. Arrows show a higher number of microaneurysms seen on FA in comparison with AngioPlex and AngioVue. $3 \times 3 \mathrm{~mm}$ angiograms from AngioPlex (E) and AngioVue (F) are overlaid with grid replicating the central subfield and inner ring of the ETDRS grid. 

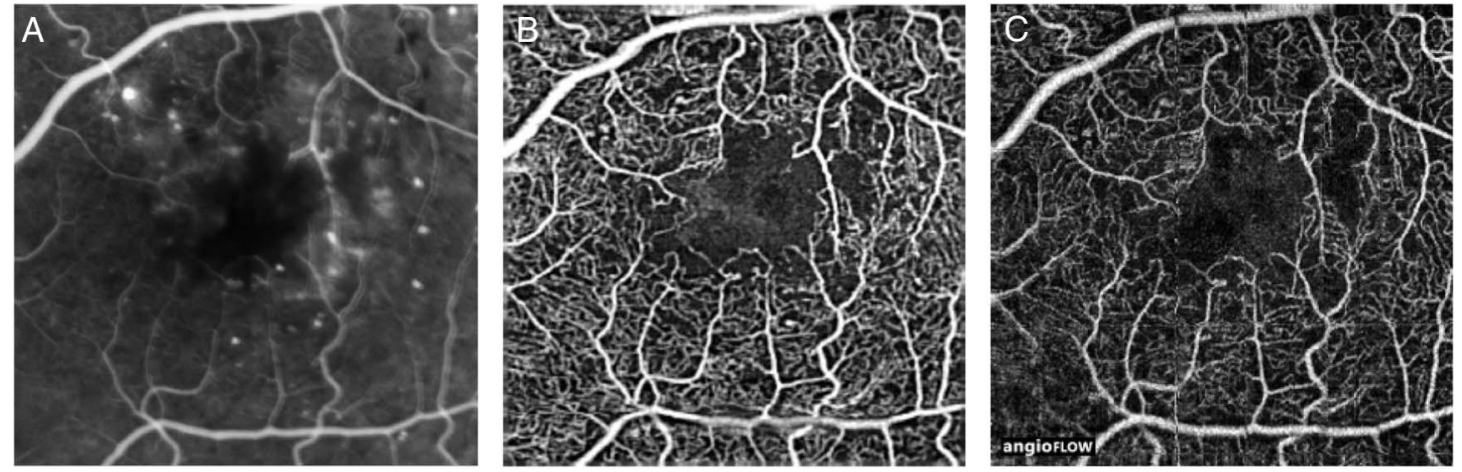

Figure 2 Comparison of fluorescein angiography (FA) with optical coherence tomography angiography (OCTA) images for the superficial retinal layer (AngioPlex and AngioVue) for foveal avascular zone and its outline. (A) $3 \times 3-\mathrm{mm}$ FA image of a diabetic eye showing poorly defined FAZ due to the presence of leakage from nearby microaneurysms. (B) $3 \times 3 \mathrm{~mm}$ OCT angiogram (AngioPlex) from the same eye showing detailed outline of the FAZ with adjacent areas of non-perfusion providing lower boundary for grade 3 (moderate) in central subfield (outline destroyed for one-half or more of the original circumference, but some remnants remain). (C) $3 \times 3 \mathrm{~mm}$ OCT angiogram from AngioVue showing the same characteristics observed in Zeiss AngioPlex images.

signal strength was below 60 for AngioVue or 6 for AngioPlex or if motion artefacts were present.

\section{Image arrangement for grading}

In order to evaluate and compare the images obtained from the three different devices, all the images were exported and a new layer was added to the original image (figure 1). The layer was developed using Gimp V.2.8.1 (gimp.org) and consists of two solid-line concentric circles centred on the fovea. The radius of the innermost solid-line circle corresponds to $500 \mu \mathrm{m}$, and the second circle to $1500 \mu \mathrm{m}$. This presentation replicates the inner ring of the ETDRS grid and is divided in five subfields: CSF, IR - superior, inferior, nasal and temporal. The enface images for OCTA, and $20^{\circ}$ images for FA, were loaded into Gimp. The grid was then overlaid manually by one of the authors, and the composed image graded. Areas corresponding to the subfields were analysed. FA images were magnified and cropped to match the OCTA images. No co-registration was performed.

\section{Grading}

Grading of the images was done based on an established grading protocol ${ }^{3}$ accounting for different characteristics. Several reference images were chosen for definition of the boundaries between steps in the grading scale (figures 2 and 3).
The OCT and FA angiograms were then independently evaluated by two trained readers.

The $3 \times 3 \mathrm{~mm}$ OCT angiograms from the SRL and full retina slab of both devices, and the $20^{\circ} \mathrm{FA}$ images (cropped to match the area from OCTA) were evaluated for the following characteristics: outline of FAZ, capillary loss and number of microaneurysms (MAs). The grading for FA and OCTA angiograms was performed independently to avoid bias. Arteriolar abnormalities (focal narrowing, narrowing or pruning and arteriolar contour) were not graded because the identification of these features in OCTA images was too ambiguous in many aspects: (1) due to the lack of references for the course of the arterioles and venules in the $3 \times 3 \mathrm{~mm}$ parafoveal area; (2) the specificity of the classification-only side branches that are perpendicular to parent arterioles are considered; (3) the existence of specific characteristics in FA-leakage and staining-which are not present on the OCTA angiograms. Capillary dilatation was also not evaluated due to the difficulty in detecting such feature on OCTA, and RPE defects were not evaluated because the segmentation was not performed for that layer.

The outline of FAZ was graded as follows: grade $0=$ outline normal; grade $1=$ outline not smoothly round or oval, appreciable irregularities seen, but not necessarily abnormal; grade
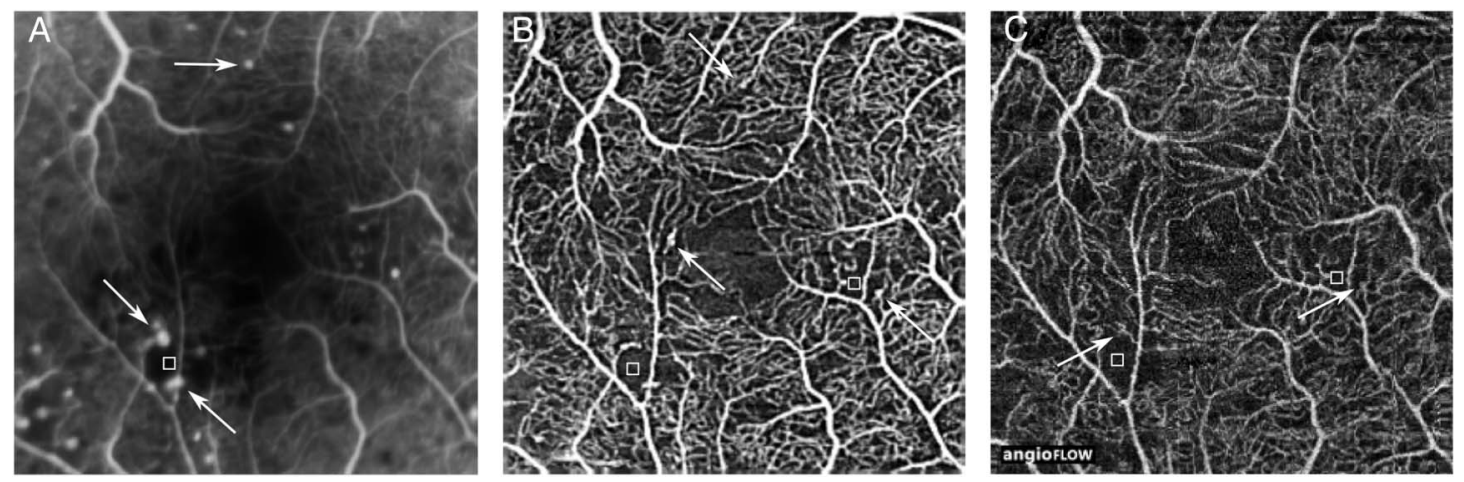

Figure 3 Comparison of fluorescein angiography (FA) with optical coherence tomography angiography (OCTA) images for the superficial retinal layer (AngioPlex and AngioVue). (A) $3 \times 3 \mathrm{~mm}$ FA image of a diabetic eye showing presence of multiple microaneurysms concentrated in the inferior and temporal subfield of the inner ring (IR) (arrows). (B) $3 \times 3 \mathrm{~mm}$ OCT angiogram (AngioPlex) showing less microaneurysms than FA images, but with capillary non-perfusion areas more defined-squares-(temporal and nasal subfield of the IR) comparing with FA image. (C) $3 \times 3 \mathrm{~mm}$ OCT angiogram (AngioVue) showing less microaneurysms than AngioPlex images. Areas of non-perfusion are also well defined, setting lower boundary for grade 2 (definitive) in all subfields of the IR. 
Table 1 Characteristics of the study sample

\begin{tabular}{ll}
\hline Patients/eyes, $\mathrm{n}$ & $26 / 50$ \\
Male/female, $\mathrm{n}$ & $16 / 10$ \\
Age, years & \\
Mean \pm SD & $64.9 \pm 7.38$ \\
$\quad$ Range & $52-76$ \\
Duration of diabetes, years & \\
$\quad$ Mean \pm SD & $18.7 \pm 6.9$ \\
Range & $7-33$ \\
Haemoglobin A1c (\%) & \\
Mean \pm SD & $7.7 \pm 1.9$ \\
Range & $6.1-14.1$ \\
ETDRS level, no. eyes (\%) & \\
10 & $8(16)$ \\
20 & $4(8)$ \\
35 & $27(54)$ \\
43 & $4(8)$ \\
47 & $4(8)$ \\
53 & $3(6)$ \\
\hline
\end{tabular}

$2=$ obviously damaged, $<180^{\circ}$; grade $3=$ obviously damaged, $>180^{\circ}$, some remnants; grade $4=$ outline totally destroyed; grade $8=$ cannot grade.

Capillary loss was graded for the CSF, and the inner ring of the ETDRS grid, divided in four subfields (superior, inferior, nasal and temporal) according to the scale: grade $0=$ absent; grade $1=$ questionable; grade $2=$ definitive (up to $25 \%$ loss in the subfield); grade $3=$ moderate $(>25 \%$ and up to $50 \%$ loss in the subfield); grade $4=$ severe ( $>50 \%$ loss in the subfield); grade $8=$ cannot grade.

$M A$ counting was also performed for all the subfields considered. MAs were defined as distinctly round, saccular or fusiform hyperfluorescent spots as described for early phase FA and on OCTA, ${ }^{20} 21$ as localised dilatations connected to the retinal capillaries.

\section{Statistics}

Original scales of outline of FAZ, and capillary loss were recoded in dummy variables for two separate analyses: graded versus cannot grade and normal versus abnormal. Outline of FAZ and capillary loss were considered graded when the original grade ranged from 1 to 4 and cannot grade when the original grade was 8 . Outline of FAZ was considered normal when the original grade ranged from 0 to 1 and abnormal when the original grade ranged from 2 to 4. Capillary loss was considered normal when the original grade was 0 and abnormal when the original grade ranged from 2 to 4 . Grades questionable or cannot grade were excluded from the normal versus abnormal analysis. Exact agreement and Cohen's $\kappa$ coefficient were calculated to assess the agreement between graders and between methods. Categorical variables are summarised with percentages and numerical variables with mean \pm SD.

\section{RESULTS}

A total of 50 eyes from 26 subjects with mean age of 65 (SD 7) with DR were imaged using FA and OCTA. A summary of the characteristics of the subjects and eyes, and the stage of DR is shown in table 1.

\section{Outline of FAZ}

For outline of FAZ in FA, the percentage of non-gradable images was $29.0 \%$. There was exact agreement for $78 \%$ of the eyes graded ( $\kappa$ 0.712); when using graded versus cannot grade, complete agreement increased to $94 \%$ ( $\kappa$ 0.854); for normal versus abnormal analysis, the percentage of agreement was $88 \%$ ( $\kappa 0.757)$.

In the AngioVue, the percentage of non-gradable images was $12.0 \%$. The percentage of agreement was $72 \%$ of the eyes graded ( $\kappa$ 0.629); when using graded versus cannot grade analysis, the complete agreement was $100 \%(\kappa 1.000)$; for normal versus abnormal analysis, the percentage was $88 \%$ ( $\mathrm{0.760}$ ).

In the AngioPlex, the percentage of non-gradable images was $3.0 \%$. The percentage of agreement was $76 \%$ of the eyes graded ( $\kappa$ 0.659); when using graded versus cannot grade analysis, the complete agreement was $98 \%$ ( $\kappa$ 0.657); for normal versus abnormal analysis, the percentage was $87 \%$ ( $\kappa 0.746)$.

For method analysis, when comparing for gradable/nongradable images, the percentage of agreement was $72.0 \%$ for FA versus AngioPlex, 71.0\% for FA versus AngioVue and $85.0 \%$ for AngioPlex versus AngioVue.

On the other hand, when comparing normal versus abnormal features, the percentage of definitive abnormal features found was superior in both OCTA devices in comparison with FA: 29.0\% for FA, 43\% for AngioPlex and 33.0\% for AngioVue.

The grading using the full retina slab showed a decrease of $2.0 \%$ for AngioVue and $4.0 \%$ for AngioPlex in the percentage of cases classified as abnormal in comparison with the SRL analysis.
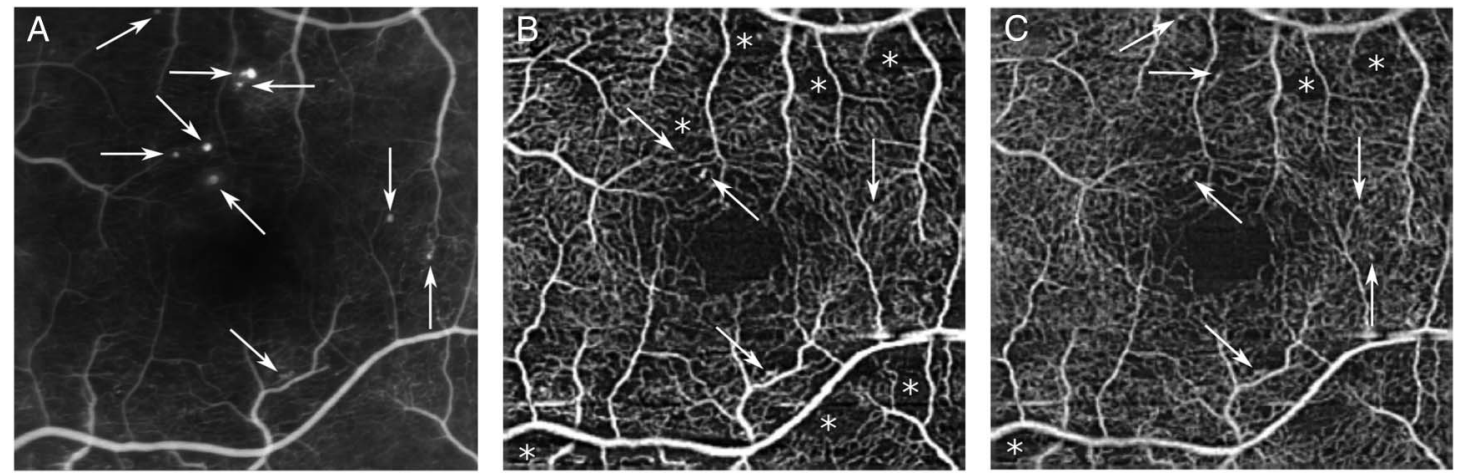

Figure 4 Comparison of fluorescein angiography (FA) images with superficial retinal layer (SRL) and full retina slab images for AngioPlex. (A) $3 \times 3$ $\mathrm{mm}$ FA image of a diabetic left eye showing presence of microaneurysms concentrated in the superior and temporal subfield of the IR (arrows). (B) $3 \times 3 \mathrm{~mm}$ OCT angiogram of the SRL (AngioPlex) showing less microaneurysms than FA images, but with capillary non-perfusion areas more defined - stars - (superior and inferior subfield of the IR) comparing with FA image. (C) $3 \times 3 \mathrm{~mm}$ OCT angiogram of the full retina slab (AngioPlex) showing more microaneurysms than in SRL images (arrows). In the full retina slab, the areas of non-perfusion are now less defined. 


\section{Capillary loss: CSF}

For capillary loss in the CSF using FA images, the percentage of non-gradable images was $25.0 \%$. Intergrader agreement for capillary loss-CSF was lower than it was for outline of FAZ: the exact agreement was 50\% ( $\kappa 0.363$ ); when using graded versus cannot grade, the complete agreement increased to $90 \%$ ( $\kappa$ 0.736); for normal versus abnormal analysis, the percentage of agreement was $74 \%$ ( $\kappa 0.137)$.

In the AngioVue, the percentage of non-gradable images was $11.0 \%$. The percentage of exact agreement was $62 \%$ of the eyes graded ( $\kappa$ 0.496); when using graded versus cannot grade analysis, the complete agreement was 98\% ( $\kappa$ 0.898); for normal versus abnormal analysis, the percentage was $82 \%$ ( $\kappa 0.443$ ).

In the AngioPlex, the percentage of non-gradable images was $0.0 \%$. The percentage of exact agreement was $56 \%$ of the eyes graded ( $\kappa$ 0.412); when using graded versus cannot grade analysis, the complete agreement was $100 \%$; for normal versus abnormal analysis, the percentage was $72 \%(\kappa 0.306)$.

For method agreement analysis, for gradable/non-gradable images, the percentage of agreement was $75.0 \%$ for FA versus AngioPlex, $72.0 \%$ for FA versus AngioVue and $89.0 \%$ for AngioPlex versus AngioVue.

When comparing normal versus abnormal features, the percentage of definitive abnormal features found was $38.0 \%$ for FA, 50.0\% for AngioPlex and 40.0\% for AngioVue.

For capillary loss in the CSF analysis of the full retina slab showed a decrease of $12.0 \%$ for both AngioVue and AngioPlex, in the percentage of cases classified as abnormal when analysing the SRL.

\section{Capillary loss: inner ring (parafoveal)}

For capillary loss, the analysis of the four subfields of the inner ring of the ETDRS grid corresponding to parafoveal region was aggregated for better discrimination and analysis. Using FA images, the percentage of non-gradable images was $12.5 \%$. Intergrader agreement for capillary loss-inner ring, was 59.0\% ( $\kappa$ 0.411) for exact agreement of the characteristics; when using graded versus cannot grade, the agreement increased to $92.0 \%$ ( $\kappa$ 0.606); for normal versus abnormal analysis, the percentage of agreement was 70.0\% ( $\kappa 0.415)$.

In the AngioVue, the percentage of non-gradable images was $11.5 \%$. The percentage of exact agreement was $75 \%$ of the eyes graded ( $\kappa$ 0.655); when using graded versus cannot grade analysis, the agreement was 98\% ( $\kappa$ 0.924); for normal versus abnormal analysis, the percentage was $87 \%$ ( $\kappa 0.733$ ).

In the AngioPlex, the percentage of non-gradable images was $0.5 \%$. The percentage of exact agreement was $74 \%$ of the eyes graded ( $\kappa$ 0.612); when using graded versus cannot grade analysis, the complete agreement was 99\%; for normal versus abnormal analysis, the percentage was $87 \%$ ( $\kappa 0.748)$.

For method agreement analysis, when comparing FA versus OCTA for gradable/non-gradable images, the results showed higher percentages in comparison with the ones obtained for the CSF: the percentage of agreement was $87.7 \%$ for FA versus AngioPlex, $81.0 \%$ for FA versus AngioVue and $88.7 \%$ for AngioPlex versus AngioVue.

When comparing normal versus abnormal features, the percentage of definitive abnormal features found was $15.0 \%$ for FA; 35.5\% for AngioPlex and 24.5\% for AngioVue.

For capillary loss in the IR analysis of the full retina slab, the percentage of cases classified as abnormal decreased in comparison with the SRL analysis by $5.0 \%$ versus the AngioVue, and by $19.0 \%$ versus the AngioPlex. An example of this trend can be observed in figure 4.

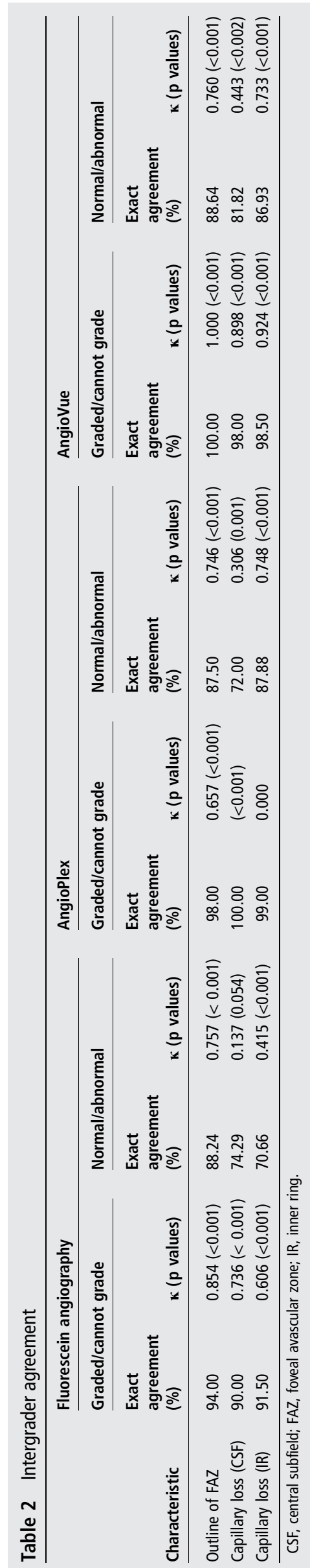




\section{MAs counting}

The visualisation and counting of the MAs was compared between the FA images and in both OCT angiography methods looking at the SRL and full retina slab. A higher number of MAs were found in FA images compared with both OCTA devices in the SRL and full retina slab. For FA the mean number

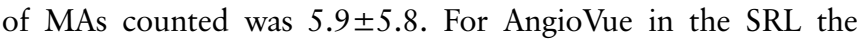
value was $1.0 \pm 1.7$, and for full retina slab the mean number

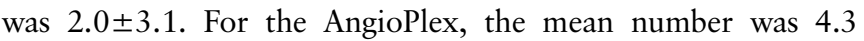
\pm 4.2 in the SRL and $5.8 \pm 6.3$ in the full retina slab. The number of MAs detected in the OCTA full retina slab comparing the MAs detected in FA was $34.0 \%$ for AngioVue and 98.0\% for AngioPlex.

The agreement between graders for MAs counting was satisfactory: for FA the exact agreement was 68\% ( $\kappa 0.424)$, for AngioPlex the exact agreement was 64\% ( $\kappa$ 0.480) and for AngioVue the agreement was 74\% ( $\kappa 0.552$ ).

Table 2 shows a summary of percentage of agreement and $\kappa$ values for grade versus non-gradable images, and for normal versus abnormal agreement between graders for all characteristics graded, for FA and OCTA.

Tables 3 and 4 summarises percentages of equipment agreement and $\kappa$ values for several characteristics in comparison with FA/OCTA for both graders.

\section{DISCUSSION}

In this study, we tried to replicate the systematic assessment proposed by the ETDRS Research Group for the analysis of FA images. Comparison between FA images and OCT angiograms was performed by two graders according to the severity of different characteristics found in the angiograms of patients with different DR levels. The percentage of non-gradable images was larger on FA images in comparison with both OCTA devices. The image quality of FA is clearly affected by the contrast, degree of pigmentation and media opacity ${ }^{22}$ and also for early leakage and superimposition of all perfused layers and choroidal blood vessels. ${ }^{21}$ On the other hand, OCTA images are affected by eye motion, scan speed, media opacity and segmentation errors that can cause several artefacts that may cause misinterpretation of the OCTA data. ${ }^{23}$

There are numerous differences between AngioVue and AngioPlex that could lead to differences in the gradability observed in this study. The two devices use different algorithms to construct the flow contrast image, but the comparability of good images suggests that this image construction algorithm is not the source of differences in gradability. The two devices have different resolution $(304 \times 304$ A-scans for AngioVue and $245 \times 245$ for AngioPlex), but this would presumably favour AngioVue. AngioPlex has four repeated B-scans while AngioVue has two, which may make the AngioPlex result more robust. AngioPlex is based on a single scan, which uses live tracking to reduce motion artefacts as discussed in the Material and methods section. AngioVue is based on the combination of two orthogonally acquired scans to reduce the effects of motion. In both cases, there is a combination of multiple datasets to improve robustness and a motion correction algorithm to reduce motion artefacts. In the case of AngioVue, if neither of the two scans contains relevant information, then some artefacts will remain, and this may lead to some of the issues with ungradability. Both methods will be subject to issues with the media of the eye. Our experience suggests some advantage in time to acquire the AngioPlex scan, which may affect the impact of drying of the cornea on the quality of the image. Thus, the most likely contributors to the improved gradability of AngioPlex images are the benefits of live eye tracking as opposed to postacquisition motion correction as well as the benefits of a faster chin time in overall image quality. In this sample, the visualisation and classification of the FAZ, and the identification of areas of capillary dropout in the parafoveal area, showed promising results with OCTA angiograms when focusing the

Table 3 Grader 1

\begin{tabular}{|c|c|c|c|c|c|c|c|c|}
\hline \multirow[b]{3}{*}{ Characteristic } & \multicolumn{4}{|c|}{ Fluorescein angiography/AngioPlex } & \multicolumn{4}{|c|}{ Fluorescein angiography/AngioVue } \\
\hline & \multicolumn{2}{|c|}{ Graded/cannot grade } & \multicolumn{2}{|l|}{ Normal/abnormal } & \multicolumn{2}{|c|}{ Graded/cannot grade } & \multicolumn{2}{|l|}{ Normal/abnormal } \\
\hline & $\begin{array}{l}\text { Exact } \\
\text { agreement (\%) }\end{array}$ & $\kappa$ ( $p$ values) & $\begin{array}{l}\text { Exact } \\
\text { agreement (\%) }\end{array}$ & $\kappa$ ( $p$ values) & $\begin{array}{l}\text { Exact } \\
\text { agreement (\%) }\end{array}$ & $\kappa$ ( $p$ values) & $\begin{array}{l}\text { Exact } \\
\text { agreement (\%) }\end{array}$ & $\kappa$ ( $p$ values) \\
\hline Outline of FAZ & 70.00 & $0.083(0.070)$ & 82.35 & $0.650(<0.001)$ & 68.00 & $0.118(0.157)$ & 87.10 & $0.729(<0.001)$ \\
\hline Capillary loss (CSF) & 70.00 & 0.000 (n.а.) & 97.14 & $0.000(0.500)$ & 68.00 & $0.058(0.304)$ & 90.63 & $0.368(0.004)$ \\
\hline Capillary loss (IR) & 85.50 & 0.000 (n.a.) & 75.44 & $0.503(<0.001)$ & 79.00 & $0.043(0.266)$ & 71.43 & $0.427(<0.001)$ \\
\hline
\end{tabular}

$\mathrm{p}$ Values are calculated from a McNemar test.

CSF, central subfield; FAZ, foveal avascular zone; IR, inner ring.

Table 4 Grader 2

\begin{tabular}{|c|c|c|c|c|c|c|c|c|}
\hline \multirow[b]{3}{*}{ Characteristic } & \multicolumn{4}{|c|}{ Fluorescein angiography/AngioPlex } & \multicolumn{4}{|c|}{ Fluorescein angiography/AngioVue } \\
\hline & \multicolumn{2}{|c|}{ Graded/cannot grade } & \multicolumn{2}{|l|}{ Normal/abnormal } & \multicolumn{2}{|c|}{ Graded/cannot grade } & \multicolumn{2}{|l|}{ Normal/abnormal } \\
\hline & $\begin{array}{l}\text { Exact } \\
\text { agreement (\%) }\end{array}$ & $\kappa$ ( $p$ values) & $\begin{array}{l}\text { Exact } \\
\text { agreement (\%) }\end{array}$ & $\kappa$ ( $p$ values) & $\begin{array}{l}\text { Exact } \\
\text { agreement (\%) }\end{array}$ & $\kappa$ ( $p$ values) & $\begin{array}{l}\text { Exact } \\
\text { agreement (\%) }\end{array}$ & $\kappa$ (p values) \\
\hline Outline of FAZ & 74.00 & $0.068(0.215)$ & 77.78 & $0.544(0.001)$ & 74.00 & $0.181(0.077)$ & 70.59 & $0.411(<0.007)$ \\
\hline Capillary loss (CSF) & 80.00 & 0.000 (n.a.) & 75.00 & $0.431(0.003)$ & 76.00 & $0.117(0.192)$ & 69.44 & $0.220(0.088)$ \\
\hline Capillary loss (IR) & 90.00 & $0.074(0.029)$ & 68.16 & $0.371(<0.001)$ & 81.00 & $0.030(0.332)$ & 57.23 & $0.160(0.018)$ \\
\hline
\end{tabular}


analysis on the SRL. We have found that when analysing the full retina slab, areas graded as non-perfused in the SRL analysis become less well-defined and more difficult to identify. The whole retina analysis shows less sensitivity to capillary dropout in comparison with the one obtained for the SRL. Nonetheless, it is important to mention that for the features that were graded, even with the full retina slab, OCTA shows better discrimination of central and parafoveal microvasculature than FA, for the capillary dropout and FAZ disruption analysis.

On the other hand, the full retina slab allowed viewing more MA-like features than in the SRL analysis. The number of MAs detected in the analysis performed using the OCTA full retina slab was clearly closer to the MAs identified in FA. We can conclude that, when looking for number of MAs using OCTA and looking for comparison with FA, it may be appropriate to use the full retina slab.

The intergrader agreement was moderate to substantial in several features. Other characteristics were not assessed with OCTA such as arteriolar abnormalities and capillary dilatation, particularly because in this study, most eyes had only mild nonproliferative diabetic retinopathy. However, experience is required, and for this training it is important to have reference images with and without pathology to evaluate and discriminate alterations found in DR.

It is noteworthy that several attempts have been made with apparent success, in quantifying the degree of retinal capillary dropout in the central macular area 2224 Our group has been working in this area, and the initial results are very promising, allowing separation between healthy eyes and eyes with mild DR. $^{25}$

FA and OCTA offer different perspectives for analysis of the retinal vasculature. FA has been around for some time now, and its interpretation is well established, whereas OCTA is just beginning and its results need to be discussed and better understood. Agreement on definition and characterisation of abnormalities detected is crucial. OCTA offers for the first time improved definition of the superficial and deep retinal capillary nets in the clinical setting. Analysis of the full retina slab on OCTA examinations may help in the transition between FA and OCTA.

Limitations of this study include the small number of patients and eyes graded. The grading was also performed only by two graders, and areas of disagreement were not reviewed in order to achieve uniformity.

In conclusion, OCTA allows better discrimination of the CSF and parafoveal macular microvasculature than FA, especially for FAZ disruption and capillary dropout. OCTA appears to be more sensitive and more reliable than FA in a clinical environment, allowing detailed morphological evaluation of the central microvascular perfusion changes. In DR, characterisation of the central microvascular perfusion changes is considered particularly relevant regarding prognosis for vision loss and progression to late-stage proliferative retinopathy.

Contributors MS, CN, MD, MAC and JC-V researched data and wrote and edited the manuscript. MS, CN, IPM, IP, CS, MAC, TS, MD and JCV researched data, contributed to the discussion and review the manuscript. MAC analysed the data, contributed to the discussion and reviewed/edited the manuscript. MS, CN, MAC, $\mathrm{MD}$ and JCV contributed to the study design, discussion, review the manuscript and are the guarantors of this work and, as such, had full access to all the data in the study and take responsibility for the integrity of the data and the accuracy of the data analysis.

Competing interests JC-V reports grants from Carl Zeiss Meditec, outside the submitted work; and José Cunha-Vaz is consultant for Alimera Sciences, Allergan,
Bayer, Gene Signal, Novartis, Pfizer, Precision Ocular, Roche, Sanofi-Aventis, Vifor Pharma and Carl Zeiss Meditec. MD reports employment from Carl Zeiss Meditec.

Patient consent Obtained.

Ethics approval National Ethics Committee for Clinical Research.

Provenance and peer review Not commissioned; externally peer reviewed.

\section{REFERENCES}

1 Novotny $\mathrm{H}$, Alvis DL. A method of photographing fluorescence in circulating blood in the human retina. Circulation 1961;24:82-6.

2 Gass JD, Sever RJ, Sparks D, et al. A combined technique of fluorescein funduscopy and angiography of the eye. Arch Ophthalmol 1967;78:455-61.

3 Anonymous. Classification of diabetic retinopathy from fluorescein angiograms. ETDRS report number 11. Early Treatment Diabetic Retinopathy Study Research Group. Ophthalmology 1991;98:807-22.

4 Wong K. Diabetic macular edema. In: Diabetic retinopathy: evidence-based management. D J Browning, Ed. 2010:141-202.

5 Early Treatment Diabetic Retinopathy Study Research Group. Fluorescein angiographic risk factors for progression of diabetic retinopathy. ETDRS report number 13. Early Treatment Diabetic Retinopathy Study Research Group. Ophthalmology 1991;98:834-40

6 Kylstra J, Brown J, Jaffe G, et al. The importance of fluorescein angiography in planning laser treatment of diabetic macular edema. Ophthalmology 1999;106:2068-73.

7 Kwiterovich KA, Maguire MG, Murphy RP, et al. Frequency of adverse systemic reactions after fluorescein angiography. Results of a prospective study. Ophthalmology 1991:98:1139-42.

8 Fingler J, Schwartz D, Yang C, et al. Mobility and transverse flow visualization using phase variance contrast with spectral domain optical coherence tomography. Opt Express 2007; 15:12636-53.

9 Fingler J, Readhead C, Schwartz DM, et al. Phase-contrast OCT imaging of transverse flows in the mouse retina and choroid. Investig Ophthalmol Vis Sci 2008;49:5055-9.

$10 \mathrm{Yu} \mathrm{L}$, Chen Z. Doppler variance imaging for three-dimensional retina and choroid angiography. J Biomed Opt 2012;15:16029.

11 Liu G, Qi W, Yu L, et al. Real-time bulk-motion-correction free Doppler variance optical coherence tomography for choroidal capillary vasculature imaging. Opt Express 2011;19:3657-66.

12 Jia Y, Tan 0, Tokayer J, et al. Split-spectrum amplitude-decorrelation angiography with optical coherence tomography. Opt Express 2012;20:4710-25.

13 Kim DY, Fingler J, Werner JS, et al. In vivo volumetric imaging of human retinal circulation with phase-variance optical coherence tomography. Biomed Opt Express 2011;2:1504-13.

14 An L, Shen TT, Wang RK. Using ultrahigh sensitive optical microangiography to achieve comprehensive depth resolved microvasculature mapping for human retina. J Biomed Opt 2011;16:106013.

15 Wang RK, An L, Francis P, et al. Depth-resolved imaging of capillary networks in retina and choroid using ultrahigh sensitive optical microangiography. Opt Lett 2010;35:1467.

16 Wang RK, Jacques SL, Ma Z, et al. Three dimensional optical angiography. Opt Express 2007:15:4083.

17 Schwartz DM, Fingler J, Kim DY, et al. Phase-variance optical coherence tomography: a technique for noninvasive angiography. Ophthalmology 2014;121:180-7.

18 Mendis KR, Balaratnasingam C, Yu P, et al. Correlation of histologic and clinical images to determine the diagnostic value of fluorescein angiography for studying retinal capillary detail. Investig Ophthalmol Vis Sci 2010;51:5864-9.

$19 \mathrm{Hu}$ J, Gottlieb CB, Barajas DJ, et al. Improved repeatability of retinal thickness measurements using line-scan ophthalmoscope image-based retinal tracking. Ophthalmic Surg Lasers Imaging Retin 2015;46:310-14.

20 Ishibazawa A, Nagaoka T, Takahashi A, et al. Optical coherence tomography angiography in diabetic retinopathy: a prospective pilot study. Am J Ophthalmol 2015:60:35-44.e1.

21 Couturier A, Bonnin S, Erginay ALI, et al. Capillary plexus anomalies in diabetic retinopathy on optical coherence tomography. Retina 2015:2384-91.

22 Hwang TS, Gao SS, Liu L, et al. Automated quantification of capillary nonperfusion using optical coherence tomography angiography in diabetic retinopathy. JAMA Ophthalmol 2016;134:367-73.

23 Spaide RF, Fujimoto JG, Waheed NK. Image artifacts in optical coherence tomography angiography. Retina 2015;35:2163-80.

24 Agemy SA, Scripsema NK, Shah CM, et al. Retinal vascular perfusion density mapping using optical coherence tomography angiography in normals and diabetic retinopathy patients. Retina 2015:2353-63.

25 Durbin MK, Soares M, An L, et al. Methods for quantification of retinal microvascular density in cirrus angioplex oct angiography (octa) images. Invest Ophthalmol Vis Sci 2016:57:5958. 